\title{
Influence of endogenous NEFA on beta cell function in humans
}

\author{
Eleni Rebelos ${ }^{1} \cdot$ Marta Seghieri $^{1} \cdot$ Andrea Natali $^{1} \cdot$ Beverly Balkau $^{2} \cdot$ \\ Alain Golay ${ }^{3}$ - Pier Marco Piatti ${ }^{4}$ - Nebojsa M. Lalic ${ }^{5}$. Markku Laakso ${ }^{6}$. \\ Andrea $\mathrm{Mari}^{7} \cdot$ Ele Ferrannini ${ }^{1,8}$
}

Received: 8 April 2015 / Accepted: 15 June 2015 /Published online: 10 July 2015

(C) Springer-Verlag Berlin Heidelberg 2015

\begin{abstract}
Aims/hypothesis It is a commonly held view that chronically elevated NEFA levels adversely affect insulin secretion and insulin action (lipotoxicity). However, the effect of NEFA on beta cell function has only been explored using acute NEFA elevations. Our aim was to analyse the relationship between endogenous NEFA levels and beta cell function.

Methods In 1,267 individuals followed-up for 3 years, we measured insulin sensitivity (by clamp) and beta cell function (by C-peptide modelling during OGTT and as the acute insulin response [AIR] to IVGTT).

Results At baseline, both fasting and insulin-suppressed NEFA levels were higher across glucose tolerance groups, while insulin sensitivity was lower, insulin output was higher, and beta cell glucose sensitivity and AIR were lower
\end{abstract}

Ele Ferrannini

ferranni@ifc.cnr.it

1 Department of Clinical \& Experimental Medicine, University of Pisa, Via Roma, 67, 56126 Pisa, Italy

2 Inserm Unit 1018, Villejuif, France

3 Division of Therapeutical Teaching for Chronic Diseases, University Hospital, Geneva, Switzerland

4 Cardio-Metabolism and Clinical Trials Unit, Department of Internal Medicine, IRCCS Ospedale San Raffaele, Milan, Italy

5 Clinic for Endocrinology, Diabetes and Metabolic Diseases, CCS, Faculty of Medicine, University of Belgrade, Belgrade, Serbia

6 Department of Medicine, University of Eastern Finland and Kuopio University Hospital, Kuopio, Finland

7 CNR Institute of Neuroscience, Padova, Italy

8 CNR Institute of Clinical Physiology, Pisa, Italy (all $p<0.0001$ ). In multiple logistic analyses adjusting for age, BMI, WHR and glucose tolerance, both fasting and insulin-suppressed NEFA levels were inversely related to insulin sensitivity, as expected (both $p<0.0001$ ). Furthermore, after adjusting for insulin sensitivity, insulin-suppressed NEFA were positively associated with total insulin output $(p=0.0042)$. In contrast, neither fasting nor insulinsuppressed NEFA were related to beta cell glucose sensitivity or AIR. At follow-up, worsening of glucose tolerance $(n=126)$ was predicted by lower insulin and beta cell glucose sensitivity. In this model, baseline NEFA were not significant predictors of progression.

Conclusions/interpretation In the non-diabetic state and in subjects with impaired glucose tolerance, circulating endogenous NEFA are not independently associated measures of beta cell function, and do not predict deterioration of glucose tolerance. Thus, in the Relationship Between Insulin Sensitivity and Cardiovascular Disease (RISC) cohort there is no evidence for beta cell lipotoxicity of endogenous total NEFA concentrations.

Keywords Beta cell function - Beta cell glucose sensitivity Glucose intolerance $\cdot$ Insulin resistance $\cdot$ Lipotoxicity $\cdot$ NEFA

\begin{tabular}{ll}
\multicolumn{2}{l}{ Abbreviations } \\
AIR & Acute insulin response to intravenous glucose \\
FFM & Fat-free mass \\
IFG & Impaired fasting glucose \\
IGT & Impaired glucose tolerance \\
IQR & Interquartile range \\
IS/AUC & Insulin secretion divided by the glucose AUC \\
ISR & Insulin secretion rate \\
NGT & Normal glucose tolerance
\end{tabular}




\section{RISC Relationship Between Insulin Sensitivity and Cardiovascular Disease \\ ssNEFA Steady-state concentrations of NEFA}

\section{Introduction}

NEFA are the major fuel substrate for almost all tissues during fasting. Their plasma levels are higher during fasting and decline after feeding owing to the antilipolytic action of insulin. NEFA levels do not correlate with the grade of adiposity [1], and are higher in women than in men due to their release primarily from subcutaneous (abdominal) rather than visceral adipose tissues [2]. Since Randle and colleagues [3] first proposed a glucose-fatty acid cycle (i.e. increased NEFA availability impairs glucose uptake), the pathophysiological effects of NEFA have been widely investigated. There is now wide consensus that insulin resistance in adipose tissue generates an excess of circulating NEFA, which in turn depress insulin sensitivity in skeletal muscle [4].

The effects of elevated NEFA concentrations on beta cell function are, however, less clear. Unger first described a negative effect of plasma NEFA on insulin secretion, for which the terms 'lipotoxicity' [5] and 'glucolipotoxicity' were coined [6]. Since then, in vitro and in vivo studies in animals and studies in humans have examined the relationship between NEFA and beta cell function, with mixed results. In vitro, addition of NEFA to perfused rat islets affects insulin release in a time-dependent fashion, with a short exposure stimulating $[7,8]$ and a prolonged exposure inhibiting insulin secretion $[9,10]$. The same response pattern is confirmed in in vivo studies in rats [11-13]. In humans, on the other hand, Boden and co-workers [14] reported that a $48 \mathrm{~h}$ lipid infusion induces an appropriate insulin secretory response in healthy individuals, which is defective in type 2 diabetic patients [15]. In contrast, Carpentier et al [16] showed that, in non-diabetic individuals, an acute (90 min) lipid infusion increases insulin secretion, and such effect disappears when the infusion is prolonged for $48 \mathrm{~h}$. The same group also reported that obese non-diabetic individuals are susceptible to the inhibitory effect of lipids on glucose-induced insulin secretion [17]. DeFronzo and colleagues [18] suggested that a sustained (4 days) exogenous infusion of NEFA inhibited glucose-induced insulin secretion in normal glucose tolerance (NGT) participants with positive family history of type 2 diabetes, whereas insulin secretion was enhanced in control participants with negative family history. Finally, an earlier study in Pima Indians reported that fasting NEFA levels are an independent risk factor for incident type 2 diabetes [19], while a more recent analysis of the same database failed to confirm this finding [20].

The heterogeneity of published studies carried out in disparate models and with different experimental protocols prompted us to ask the question, whether endogenous, i.e. circulating, NEFA bear an independent relation to beta cell function when the latter is assessed by multiple variables, and whether NEFA levels are an independent predictor of deterioration of glucose tolerance in non-diabetic individuals. To this end, we analysed the baseline and follow-up data of the Relationship Between Insulin Sensitivity and Cardiovascular Disease (RISC) study, in which 1,300 healthy non-diabetic participants received a euglycaemic-hyperinsulinaemic clamp as a direct measure of insulin sensitivity, an OGTT and an IVGTT for the assessment of various aspects of beta cell function.

\section{Methods}

Study cohort RISC is a prospective, observational, cohort study whose rationale and methodology have been published previously [21]. In brief, participants were recruited from the local population at 19 centres in 13 countries in Europe according to the following inclusion criteria: either sex, age 3060 years (stratified by sex and by age according to 10 -year age groups), BMI $17-44 \mathrm{~kg} / \mathrm{m}^{2}$ and clinically healthy. Initial exclusion criteria were treatment for obesity, hypertension, lipid disorders or diabetes, pregnancy, cardiovascular or chronic lung disease, weight change of $\geq 5 \mathrm{~kg}$ in past month, cancer (in past 5 years) and renal failure. Exclusion criteria after screening were arterial $\mathrm{BP} \geq 140 / 90 \mathrm{mmHg}$, fasting plasma glucose $\geq 7.0 \mathrm{mmol} / \mathrm{l}, 2 \mathrm{~h}$ plasma glucose (on a standard $75 \mathrm{~g}$ OGTT performed in each individual) $\geq 11.0 \mathrm{mmol} / \mathrm{l}$ or known diabetes, total serum cholesterol $\geq 7.8 \mathrm{mmol} / \mathrm{l}$, serum triacylglycerols $\geq 4.6 \mathrm{mmol} / \mathrm{l}$ and electrocardiogram abnormalities. Baseline examinations included 1,538 participants receiving an OGTT. Of these, 1,267 also received a euglycaemic-hyperinsulinaemic clamp; their baseline data have been published [22].

All 1,267 participants of the baseline clamp cohort were recalled 3 years later and 1,040 (82\%) participated in the follow-up evaluation. The baseline anthropometric and metabolic characteristics of the 227 individuals who were lost to follow-up were superimposable on those of the individuals who participated (data not shown). The follow-up study included all the baseline measurements (anthropometrics, routine blood chemistry and OGTT) except for the glucose clamp.

Local ethics committee approval was obtained by each recruiting centre. Participants were given detailed written information on the study as well as an oral explanation, and they all signed a consent form.

Based on the observed changes of glucose tolerance at follow-up, participants were classified as non-progressors (i.e. NGT at both baseline and follow-up) or progressors (i.e. those stepping up along the sequences NGT $\rightarrow$ impaired fasting glucose (IFG), NGT $\rightarrow$ impaired glucose tolerance 
(IGT), NGT $\rightarrow$ type 2 diabetes, IFG $\rightarrow$ IGT, IFG $\rightarrow$ type 2 diabetes, IGT $\rightarrow$ type 2 diabetes, between baseline and follow-up).

OGTT Blood samples were taken before and at 30, 60, 90 and $120 \mathrm{~min}$ into the OGTT. Blood samples were separated into plasma and serum, aliquotted, and stored at $-80^{\circ} \mathrm{C}$ for glucose, insulin and C-peptide determination. Samples were transported on dry ice at prearranged intervals to central laboratories.

Insulin clamp On a separate day within 1 week of the OGTT, a euglycaemic-hyperinsulinaemic clamp was performed in all participants. Exogenous insulin was infused at a rate of 240 pmol min $\mathrm{m}^{-1} \mathrm{~m}^{-2}$ simultaneously with a variable $20 \%$ dextrose infusion adjusted every 5-10 min to maintain plasma glucose level within $0.8 \mathrm{mmol} / \mathrm{l}( \pm 15 \%)$ of the target glucose level $(4.5-5.5 \mathrm{mmol} / \mathrm{l})$.

IVGTT In 762 of the 1,267 participants, the acute insulin response to intravenous glucose (AIR) was measured at the end of the clamp: a glucose bolus $(0.3 \mathrm{mg} / \mathrm{kg}$ body weight $)$ was injected over $1 \mathrm{~min}$, and plasma glucose, insulin and C-peptide concentrations were measured at 2, 4, 6 and 8 min after the bolus.

Analytical procedures Plasma glucose was measured by the glucose oxidase technique. Serum insulin was measured by a specific time-resolved immunofluorometric assay (AutoDELFIA, Insulin kit, Wallac, Turku, Finland) with the following assay characteristics: detection limit $>3 \mathrm{pmol} / \mathrm{l}$, intra- and interassay variation $1.7 \%$ and $3.5 \%$, respectively. The intra- and interassay coefficient of variation was $<5$ and $<10 \%$, respectively. NEFA were assayed by a fluorimetric method (Wako, Neuss, Germany).

Data analysis Fat-free mass (FFM) was evaluated by the TANITA bioimpedance balance (Tanita International Division, UK). Fat mass was obtained as the difference between body weight and FFM. Glucose tolerance was categorised into NGT, IGT and IFG [21].

Insulin sensitivity was calculated as the $M$ value during the final $40 \mathrm{~min}$ of the $2 \mathrm{~h}$ clamp (normalised to the FFM and the mean plasma insulin concentration measured during the same interval: $\mathrm{M} / \mathrm{I}$, in units of $\mu \mathrm{mol} \mathrm{kg} \mathrm{FFM}^{-1} \mathrm{~min}^{-1}$ $\left.[\mathrm{nmol} / \mathrm{l}]^{-1}\right)$.

Because fasting NEFA show wide between- and withinsubject variability (as they are strongly related to fasting insulin and glucose levels), steady-state NEFA (ssNEFA) levels were used for the main analyses. In fact, under clamp conditions (i.e. at similar plasma insulin and glucose levels) plasma NEFA are stable in each individual during the second hour of the clamp.
Beta cell function Beta cell function was assessed from the OGTT using a model describing the relationship between insulin secretion rate (ISR, expressed in pmol min ${ }^{-1} \mathrm{~m}^{-2}$ ) and glucose concentration as the sum of two components [23, 24]. The first component represents the dependence of ISR on glucose concentration through a dose-response function relating the two variables. From the dose-response, beta cell glucose sensitivity (the slope) is calculated. The dose-response is modulated by a potentiation factor, accounting for various mechanisms (prolonged hyperglycaemia, non-glucose substrates, gastrointestinal hormones, neural modulation). The potentiation factor averages 1 during the test and expresses relative potentiation or inhibition of ISR; its excursion is quantified by the ratio between the $2 \mathrm{~h}$ and the baseline value (potentiation ratio). The second ISR component represents the dependence of ISR on the rate of change of glucose concentration and is determined by a single parameter (rate sensitivity), which is related to early insulin release [25]. The model parameters were estimated from glucose and C-peptide concentrations (using C-peptide deconvolution as previously described [26]). The integral of ISR during the whole test (total ISR) was also calculated.

With the IVGTT, the acute insulin response to intravenous glucose was expressed as the incremental insulin secretion/ glucose area ratio over the same time interval $\left(\mathrm{IS} / \mathrm{AUC}_{\mathrm{G}}\right.$, in pmol $\left.\mathrm{m}^{-2}[\mathrm{mmol} / \mathrm{l}]^{-1}\right)$. Peripheral insulin clearance rate was calculated as the ratio of nominal exogenous insulin infusion rate and steady-state plasma insulin concentrations during the clamp.

Statistical analysis Data are reported as mean $\pm \mathrm{SD}$; variables with skewed distribution are summarised as median and interquartile range (IQR) and were logarithmically transformed for use in parametric statistical testing. Group values were compared by the Mann-Whitney or the Kruskal-Wallis test for continuous variables, or the $\chi^{2}$ test for nominal variables. ANCOVA was used to adjust group comparisons for potential confounders. Simple associations were tested by Spearman $\rho$, and logistic regression was used to predict outcome. A $p$ value $\leq 0.05$ was considered statistically significant.

\section{Results}

Baseline As expected, all the anthropometric and metabolic variables (including plasma glucose, insulin, and glucagon) worsened across glucose tolerance status from NGT to IFG to IGT (Table 1). Despite their wide variability $(\sim 50 \%)$, fasting NEFA levels also increased from NGT to IGT. After partialling for age (partial $r=0.07, p<0.01$ ), BMI (partial $r=-0.03, p=\mathrm{ns}$ ), WHR (partial $r=-0.12, p<0.0001$ ) and insulin sensitivity (partial $r=-0.14, p<0.0001$ ), the difference in 
Table 1 Anthropometric and metabolic characteristics of the study participants

\begin{tabular}{lllll}
\hline & NGT & IFG & IGT & $p^{\mathrm{a}}$ \\
\hline$n(\%$ female) & $968(59)$ & $183(34)$ & $116(59)$ & $\mathrm{ns}$ \\
Age (years) & $44 \pm 8$ & $47 \pm 9$ & $46 \pm 8$ & 0.0001 \\
Familial diabetes (\%) & 23 & 36 & 48 & $<0.0001$ \\
BMI $\left(\mathrm{kg} / \mathrm{m}^{2}\right)$ & $24.9 \pm 3.8$ & $27.1 \pm 3.7$ & $27.6 \pm 4.4$ & $<0.0001$ \\
Fat mass $(\%)$ & $27 \pm 9$ & $28 \pm 9$ & $33 \pm 8$ & $<0.0001$ \\
Waist/hip $(\mathrm{cm} / \mathrm{cm})$ & $0.85 \pm 0.10$ & $0.91 \pm 0.11$ & $0.90 \pm 0.12$ & $<0.0001$ \\
Fasting glucose (mmol/l) & $4.9 \pm 0.4$ & $5.9 \pm 0.3$ & $5.3 \pm 0.6$ & $<0.0001$ \\
2 h glucose (mmol/l) & $5.3 \pm 1.1$ & $5.8 \pm 1.1$ & $8.7 \pm 0.7$ & $<0.0001$ \\
Fasting insulin (pmol/l) & $28(20)$ & $40(24)$ & $44(33)$ & $<0.0001$ \\
Fasting glucagon (pmol/l) & $8.9 \pm 4.1$ & $8.8 \pm 3.9$ & $10.4 \pm 4.8$ & $<0.0017$ \\
Fasting NEFA ( $\mu \mathrm{mol} / \mathrm{l})$ & $500(260)$ & $510(267)$ & $610(310)$ & $<0.0001$ \\
LDL-cholesterol (mmol/l) & $2.8(1.1)$ & $3.1(1.1)$ & $3.0(0.9)$ & 0.0001 \\
HDL-cholesterol (mmol/l) & $1.4(0.5)$ & $1.3(0.4)$ & $1.3(0.5)$ & 0.0001 \\
Triacylglycerols $(\mathrm{mmol} / \mathrm{l})$ & $0.87(0.55)$ & $1.13(0.72)$ & $1.18(0.86)$ & $<0.0001$ \\
\hline
\end{tabular}

Data are means $\pm \mathrm{SD}$ or median (IQR) for normally or non-normally distributed variables

${ }^{a} p$ values are from ANOVA or Kruskal-Wallis tests fasting NEFA across the three groups was still significant though attenuated (partial $r=0.09, p=0.001$ ).

During the clamp, while steady-state plasma insulin concentrations were similar across the three groups, insulin sensitivity declined significantly (Table 2). As expected, ssNEFA levels (i.e. last $40 \mathrm{~min}$ of the $2 \mathrm{~h}$ clamp) were suppressed relative to fasting NEFA ( $p<0.0001$ for each group), but such suppression decreased from NGT to IGT (from $90 \%$ for NGT to $85 \%$ for IGT, $p<0.0001$ ). After partialling for age, BMI, WHR and insulin sensitivity, ssNEFA was progressively higher across the three groups $(p=0.017)$.
During the OGTT, fasting ISR and total insulin output increased, whereas beta cell glucose sensitivity and potentiation decreased, across the three groups. During the IVGTT, the acute insulin secretory response (IS/AUC ${ }_{\mathrm{G}}$ ) decreased across groups; in the whole dataset, $\mathrm{IS} / \mathrm{AUC}_{\mathrm{G}}$ was positively correlated with beta cell glucose sensitivity from the OGTT $(\rho=0.30, p<0.0001)$.

In the whole dataset, total insulin output was directly related - and glucose sensitivity was reciprocally related - to sexspecific quartiles of $2 \mathrm{~h}$ plasma glucose concentrations (Fig. 1). In bivariate analysis, total insulin output was directly
Table 2 Insulin sensitivity and beta cell function variables

\begin{tabular}{|c|c|c|c|c|}
\hline & NGT & IFG & IGT & $p^{\mathrm{a}}$ \\
\hline Steady-state insulin $(\mathrm{pmol} / \mathrm{l})$ & 401 (119) & $398(118)$ & $421(139)$ & ns \\
\hline $\mathrm{M} / \mathrm{I}\left(\mu \mathrm{mol} \mathrm{kg} \mathrm{FFM}^{-1} \min ^{-1}[\mathrm{nmol} / 1]^{-1}\right)$ & $135(86)$ & $123(80)$ & $89(64)$ & $<0.0001$ \\
\hline MCR-I $\left(1 \min ^{-1} \mathrm{~m}^{-2}\right)$ & $0.60(0.19)$ & $0.60(0.13)$ & $0.57(0.19)$ & ns \\
\hline SsNEFA $(\mu \mathrm{mol} / 1)$ & $25(30)$ & $34(30)$ & $50(80)$ & $<0.0001$ \\
\hline \multicolumn{5}{|l|}{ OGTT } \\
\hline Fasting ISR (pmol $\min ^{-1} \mathrm{~m}^{-2}$ ) & $65(35)$ & $84(41)$ & $91(49)$ & $<0.0001$ \\
\hline Total IS $\left(\mathrm{nmol} / \mathrm{m}^{-2}\right)$ & $38(17)$ & $41(15)$ & $50(21)$ & $<0.0001$ \\
\hline Beta cell GS (pmol $\left.\min ^{-1} \mathrm{~m}^{-2}[\mathrm{mmol} / 1]^{-1}\right)$ & $121(83)$ & $98(74)$ & $66(43)$ & $<0.0001$ \\
\hline Rate sensitivity $\left(\mathrm{pmol} \mathrm{m}{ }^{-2}[\mathrm{mmol} / \mathrm{l}]^{-1}\right)$ & $833(1,368)$ & $631(869)$ & $775(774)$ & ns \\
\hline Potentiation factor (ratio) & $1.69(1.30)$ & $1.96(1.16)$ & $1.39(0.64)$ & $<0.0001$ \\
\hline \multicolumn{5}{|l|}{ IVGTT } \\
\hline $\mathrm{AUC}_{\mathrm{G}}(\mathrm{mmol} / \mathrm{l})$ & $6.9(2.7)$ & $7.5(2.8)$ & $8.0(2.7)$ & $<0.0001$ \\
\hline $\mathrm{AUC}_{\mathrm{I}}(\mathrm{pmol} / \mathrm{l})$ & $93(181)$ & $84(183)$ & $89(153)$ & ns \\
\hline IS/AUC ${ }_{\mathrm{G}}\left(\mathrm{pmol} \mathrm{m}{ }^{-2}[\mathrm{mmol} / \mathrm{l}]^{-1}\right)$ & $479(352)$ & $438(352)$ & $381(262)$ & 0.0012 \\
\hline
\end{tabular}

Data are means \pm SD or median (IQR) for normally or non-normally distributed variables

${ }^{a} p$ values are from Kruskal-Wallis test

$\mathrm{AUC}_{\mathrm{I}}$, Incremental AUC insulin; GS, glucose sensitivity; MCR-I, peripheral insulin clearance rate 

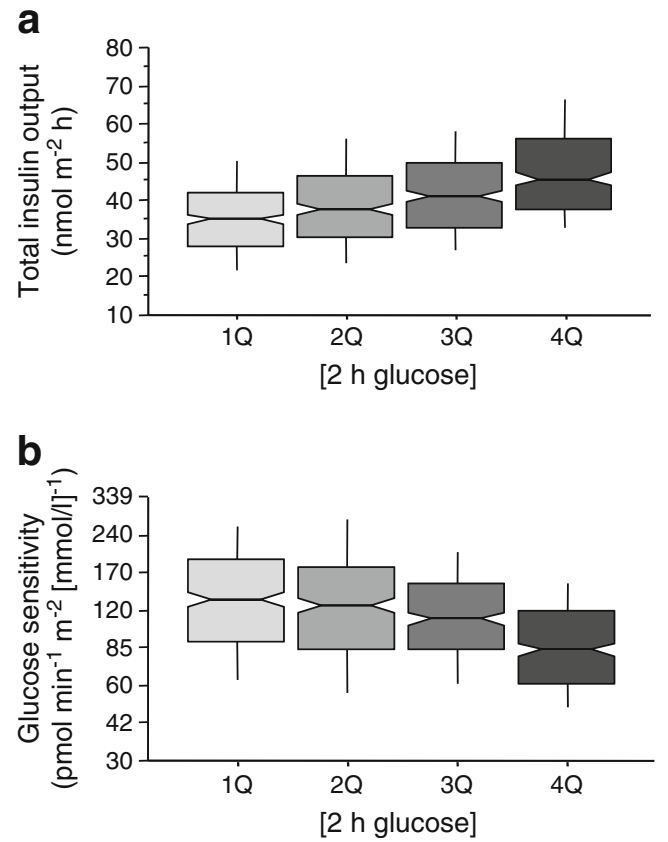

Fig. 1 Total insulin output (a) and beta cell glucose sensitivity (b) over the $2 \mathrm{~h}$ of the OGTT by quartile $(\mathrm{Q})$ of baseline $2 \mathrm{~h}$ plasma glucose concentrations $(p<0.0001)$

related to $2 \mathrm{~h}$ plasma glucose $(p<0.0001)$ and, independently, to NEFA (as sex-specific quartiles of ssNEFA) $(p<0.0001)$. In contrast, glucose sensitivity was strongly related to $2 \mathrm{~h}$ plasma glucose quartiles in an inverse manner $(p<0.0001)$ but not to ssNEFA quartiles (Fig. 2). In a multivariate model controlling for sex, age, BMI, WHR, $2 \mathrm{~h}$ plasma glucose (and also insulin
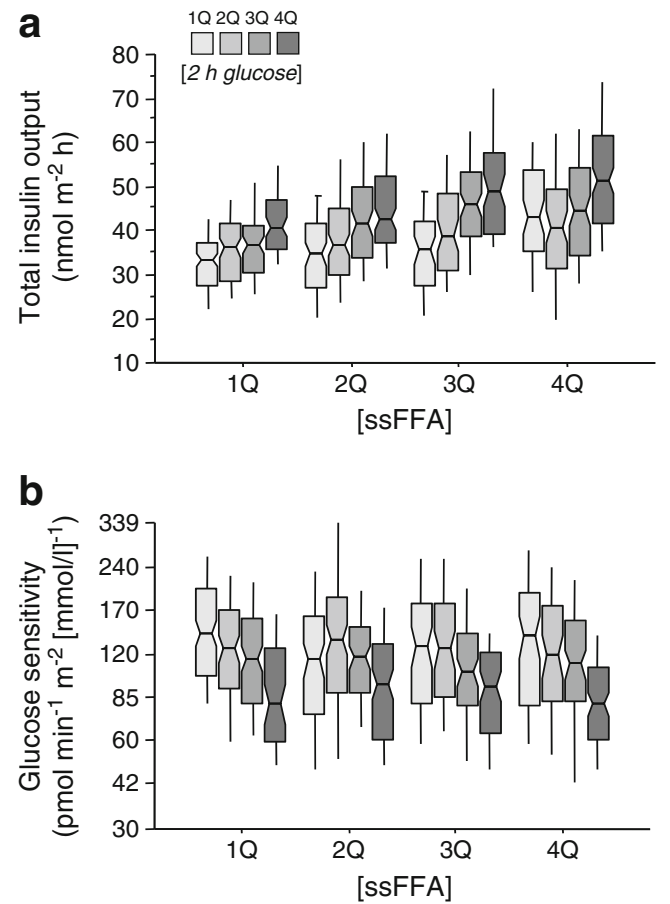

Fig. 2 Total insulin output (a) and beta cell glucose sensitivity (b) by quartiles $(\mathrm{Q})$ of baseline $2 \mathrm{~h}$ plasma glucose concentrations and ssNEFA sensitivity in the case of the beta cell variables), ssNEFA were significantly associated with insulin sensitivity (reciprocally, $p<0.0001$ ) and total insulin output (directly, $p<0.0001$ ), but not to beta cell glucose sensitivity ( $p=0.67$; Fig. 3 ). In this model, ssNEFA were reciprocally related to peripheral insulin clearance $(p<0.03)$, in line with previous work [27]. Of interest was that ssNEFA were reciprocally associated with the potentiation ratio $(p=0.03$, after adjusting for sex, age and BMI).

Of note, when in these multivariate models SSNEFA levels were replaced by the fasting NEFA concentrations, the results were similar (not shown).
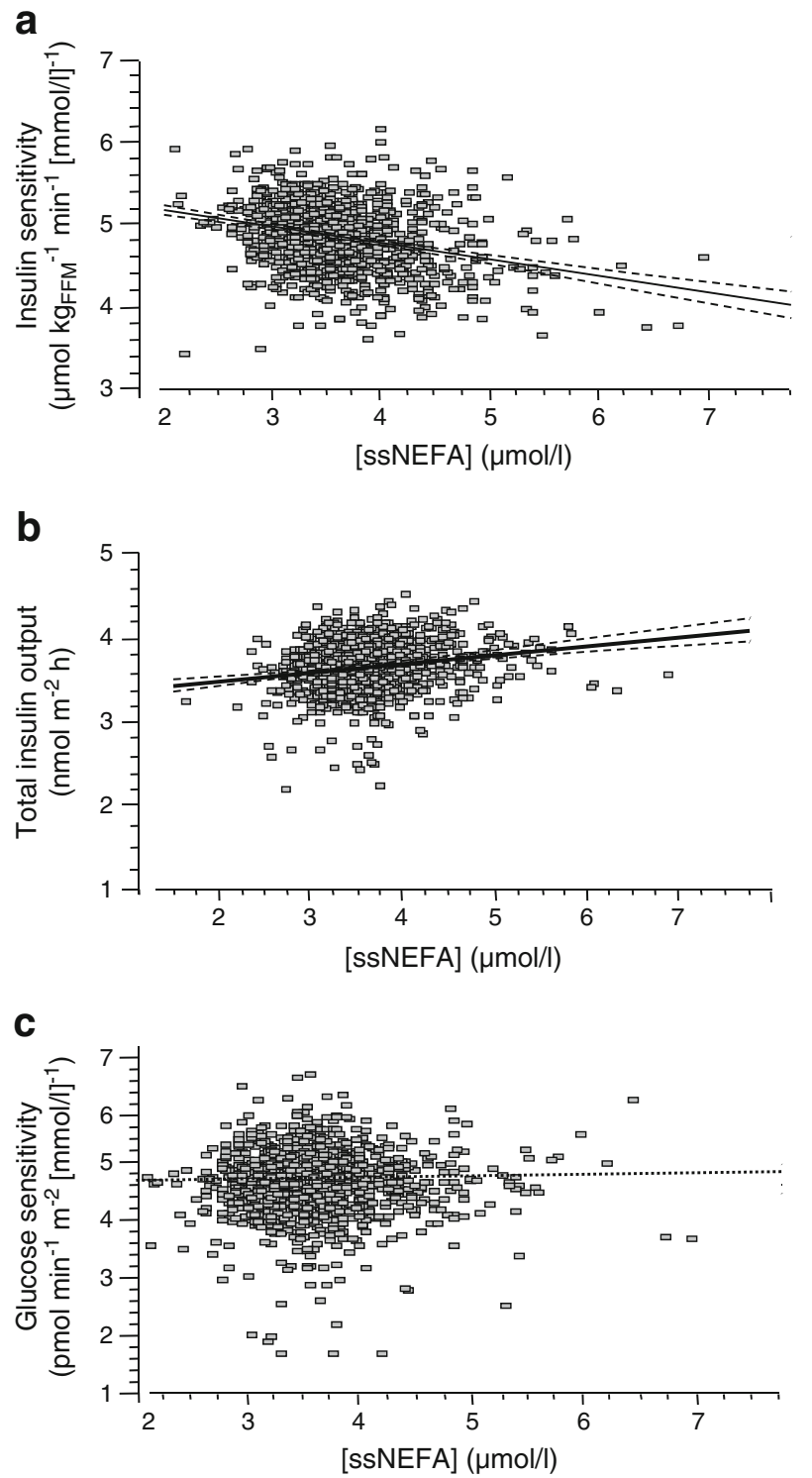

Fig. 3 Insulin sensitivity (a), total insulin output (b) and beta cell glucose sensitivity (c) as a function of ssNEFA ( $p<0.0001, p<0.0001, p=\mathrm{ns}$, respectively). The log-log relationships are adjusted for sex, age, BMI, WHR, $2 \mathrm{~h}$ plasma glucose (and also insulin sensitivity in the case of the beta cell glucose sensitivity) 
Follow-up At follow-up, 866 participants were NGT, 40 were IFG, 123 were IGT and 19 had type 2 diabetes. In total, 126 participants were progressors, i.e., their glucose tolerance was worse at follow-up than at baseline. Baseline ssNEFA, but not baseline fasting NEFA, was significantly $(p=0.02)$ higher in progressors than non-progressors (33 [35] vs 25 [30] $\mu \mathrm{mol} / 1)$. In univariate logistic regression, baseline ssNEFA predicted progression $(p<0.05)$. However, in the full prediction model, including WHR, fasting plasma glucose, insulin sensitivity and beta cell glucose sensitivity, baseline ssNEFA levels were unrelated to progression (Fig. 4).

Of note, in the baseline cohort there were 23 NGT participants ( 13 women and 10 men) with both a father and mother with type 2 diabetes. When compared with an age-, sex- and BMI-matched group of NGT participants with negative family history of type 2 diabetes, the only significant difference was in insulin sensitivity (124 [57] vs 150 [88] $\mu_{\mathrm{mol} \mathrm{kg}}{ }^{-1}$ $\left.\min ^{-1}[\mathrm{nmol} / 1]^{-1}, p<0.01\right)$, whereas all other variablesWHR, fasting and $2 \mathrm{~h}$ glucose, fasting NEFA levels, steadystate plasma insulin concentrations during the clamp and glucose sensitivity - were not significantly different. While ssNEFA levels tended to be higher in the participants with positive family history of type 2 diabetes (37 [40] vs 25 [30] $\mu \mathrm{mol} / 1, p=0.06$ ), they were not significantly related to beta cell glucose sensitivity, and the reciprocal relationship between NEFA and insulin sensitivity was similar in these two groups as was the progression rate to dysglycaemia at follow up.

\section{Discussion}

The main findings from this work were: (1) circulating NEFA levels were inversely related to insulin sensitivity; (2) NEFA were directly related to absolute insulin release; (3) NEFA were unrelated to beta cell glucose sensitivity; and (4) baseline

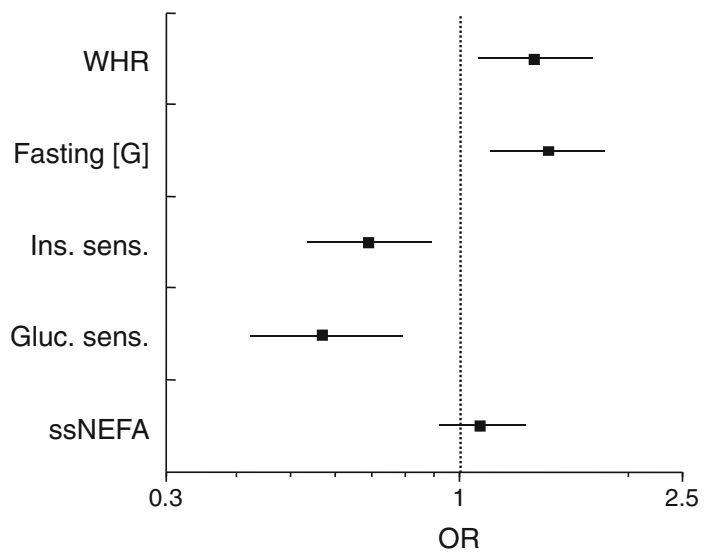

Fig. 4 Multiple logistic regression model for incident dysglycaemia. Fasting [G], fasting plasma glucose concentration; Ins. sens., insulin sensitivity on the clamp; Gluc. sens., beta cell glucose sensitivity
NEFA did not predict deterioration of glucose tolerance at follow-up. These results require specification.

First, the reciprocal relationship between endogenous NEFA and insulin sensitivity has been consistently reported in previous studies [4, 28]. In our cohort, this association was present regardless of glucose tolerance status, and was still statistically significant after adjusting for all measured confounders, including plasma glucose levels themselves. In support of the relation of NEFA to insulin action are also studies demonstrating that acutely raising plasma NEFA induces insulin resistance [29], whereas pharmacological suppression of NEFA release (e.g., with the use of acipimox) potentiates insulin-mediated glucose uptake [30].

With regard to the direct association of NEFA with absolute insulin release, the literature reports mixed results, obtained with a variety of methods, sample sizes and experimental protocols (especially for the measurement of insulin secretion). As argued by Karpe et al [31], the main difficulty is represented by the fact that fasting NEFA typically show wide betweenand within-subject variability as they are strongly related to fasting insulin and glucose levels (Table 1). In contrast, under clamp conditions (i.e. at similar plasma insulin and glucose levels) plasma NEFA are stable in each individual during the second hour of the clamp. We find that these ssNEFA levels are positively related to insulin secretion - as measured during the OGTT - independently of sex, obesity, insulin sensitivity and glucose tolerance status (Fig. 3). This finding provides strong support to the notion that endogenous NEFA normally exert a tonic stimulatory influence on insulin release. Coherent with this conclusion are the studies of Dobbins et al [32], which showed that suppressing lipolysis in fasted humans led to a $30 \%-40 \%$ inhibition of the insulin response to intravenous glucose in comparison with maintaining plasma NEFA by an Intralipid+heparin infusion. On the other hand, DeFronzo and colleagues [18] reported a small inhibitory effect on insulin release of a sustained (4 days) lipid infusion in a group of NGT participants with familial type 2 diabetes as compared with NGT participants without familial type 2 diabetes, in whom insulin release was marginally stimulated. Of note is that such differences emerged only when C-peptide concentrations were used to index insulin secretion, while plasma insulin levels were indeed similar between the two study groups. In fact, the authors attributed this disconnect to a decreased hepatic insulin clearance [18]. In our matched groups of NGT participants without or with strong family history of type 2 diabetes, we were unable to find any difference in insulin release that could not be accounted for by the degree of insulin resistance. These divergent results may be explained by the circumstance that DeFronzo and colleagues [18] used a prolonged infusion of exogenous lipids whereas we sought to establish a link between endogenous NEFA and insulin secretion. 
In contrast with the results on absolute insulin secretion, we did not detect an independent relation of NEFA to beta cell glucose sensitivity, which is the main mode of beta cell function to control glycaemia [23, 25]. We therefore conclude that the current results do not support the paradigm of lipotoxicity. The concept of lipotoxicity has emerged primarily from in vitro studies of cultured islets or beta cell lines, typically exposed to high concentrations of the saturated fatty acid, palmitate. The monounsaturated fatty acid, oleate, on the other hand, has been shown to stimulate glucose-induced insulin release, at least in some in vitro systems (e.g., cultured INS-1e cells [33]). In line with this construct, the composition of human plasma NEFA - which reflects adipose tissue and dietary fatty acid composition - shows a predominance of monounsaturated $(\sim 40 \%)$ over saturated fatty acids $(\sim 27 \%)$ [34]. Finally, to our knowledge, no human studies have measured beta cell glucose sensitivity separately from absolute insulin secretion in a large enough sample covering a wide range of BMI and insulin sensitivity, as we did in the present study.

In accordance with the cross-sectional data, we report that baseline NEFA levels did not predict incident dysglycaemia in either the NGT or IFG/IGT participants in our cohort. Of note is that a study in Pima Indians proposed that basal fasting NEFA levels predicted incident type 2 diabetes in this ethnic group [19]. However, a subsequent analysis of the same database using appropriate statistical adjustment for confounders failed to confirm the hypothesis, and concluded that chronically elevated plasma NEFA have a deleterious effect on insulin secretory capacity only in participants with IGT [20]. Likewise, in a prospective cohort from the Ely study, fasting NEFA concentrations did not predict the development of type 2 diabetes [35], while a more recent analysis of this cohort reached the opposite conclusion [36].

In summary, our data show that plasma NEFA are strongly related to insulin sensitivity in a reciprocal fashion, and are independently related to insulin secretion in a direct manner. On the other hand, NEFA are not independently associated with beta cell glucose sensitivity nor do they predict incident dysglycaemia independently of its main determinants (beta cell glucose insensitivity and insulin resistance). It is important to emphasise that this conclusion applies to endogenous circulating NEFA as measured in a cohort of white participants. We cannot rule out that NEFA from other sources, such as the lipid emulsions used in acute infusion experiments or from test diets, may show a different relation to beta cell function or insulin sensitivity. Also, it is possible that lipid species that circulate at very low concentrations or are secreted in response to fat ingestion [37] may act as signal molecules to influence some aspect of beta cell function.
Funding The RISC Study was supported by EU Grant QLG1-CT2001-01252.

Duality of interest The authors declare that there is no duality of interest associated with this manuscript.

Contribution statement ER, MS, AN, BB, AG, PP, NL, ML and AM acquired and analysed data and literature, and drafted the manuscript. AM also performed the mathematical modelling. EF conceived the design and reviewed the manuscript. All authors approved the final version of the manuscript. EF is the guarantor of this work.

\section{References}

1. Ferrannini E, Camastra S, Coppack SW, Fliser D, Golay A, Mitrakou A (1997) Insulin action and non-esterified fatty acids. The European Group for the Study of Insulin Resistance (EGIR). Proc Nutr Soc 56:753-761

2. Lafontan M, Langin D (2009) Lipolysis and lipid mobilization in human adipose tissue. Prog Lipid Res 48:275-797

3. Randle PJ, Garland PB, Hales CN, Newsholme EA (1963) The glucose fatty-acid cycle. Its role in insulin sensitivity and the metabolic disturbances of diabetes mellitus. Lancet 1:785-789

4. Samuel VT, Petersen KF, Shulman GI (2010) Lipid-induced insulin resistance: unravelling the mechanism. Lancet 375:2267-2277

5. Unger RH (1995) Lipotoxicity in the pathogenesis of obesitydependent NIDDM. Genetic and clinical implications. Diabetes 44:863-870

6. Briaud I, Harmon JS, Kelpe CL, Segu VB, Poitout V (2001) Lipotoxicity of the pancreatic beta-cell is associated with glucosedependent esterification of fatty acids into neutral lipids. Diabetes 50:315-321

7. McGarry JD (2002) Banting lecture 2001: dysregulation of fatty acid metabolism in the etiology of type 2 diabetes. Diabetes 51:718

8. Warnotte C, Gilon P, Nenquin M, Henquin JC (1994) Mechanisms of the stimulation of insulin release by saturated fatty acids. A study of palmitate effects in mouse beta-cells. Diabetes 43:703-711

9. Zhou YP, Grill VE (1994) Long-term exposure of rat pancreatic islets to fatty acids inhibits glucose-induced insulin secretion and biosynthesis through a glucose fatty acid cycle. J Clin Invest 93: 870-876

10. Elks ML (1993) Chronic perifusion of rat islets with palmitate suppresses glucose-stimulated insulin release. Endocrinology 133: 208-214

11. Stein DT, Esser V, Stevenson BE et al (1996) Essentiality of circulating fatty acids for glucose-stimulated insulin secretion in the fasted rat. J Clin Invest 97:2728-2735

12. Sako Y, Grill VE (1990) A 48-hour lipid infusion in the rat timedependently inhibits glucose-induced insulin secretion and B cell oxidation through a process likely coupled to fatty acid oxidation. Endocrinology 127:1580-1589

13. Mason TM, Goh T, Tchipashvili Vet al (1999) Prolonged elevation of plasma free fatty acids desensitizes the insulin secretory response to glucose in vivo in rats. Diabetes 48:524-530

14. Boden G, Chen X, Rosner J, Barton M (1995) Effects of a 48-h fat infusion on insulin secretion and glucose utilization. Diabetes 44: $1239-1242$

15. Boden G, Chen X (1999) Effects of fatty acids and ketone bodies on basal insulin secretion in type 2 diabetes. Diabetes 48:577-583

16. Carpentier A, Mittelman SD, Lamarche B, Bergman RN, Giacca A, Lewis GF (1999) Acute enhancement of insulin secretion by FFA in 
humans is lost with prolonged FFA elevation. Am J Physiol 276: E1055-E1066

17. Carpentier A, Mittelman SD, Bergman RN, Giacca A, Lewis GF (2000) Prolonged elevation of plasma free fatty acids impairs pancreatic beta-cell function in obese nondiabetic humans but not in individuals with type 2 diabetes. Diabetes 49:399-408

18. Kashyap S, Belfort R, Gastaldelli A et al (2003) A sustained increase in plasma free fatty acids impairs insulin secretion in nondiabetic subjects genetically predisposed to develop type 2 diabetes. Diabetes 52:2461-2474

19. Paolisso G, Tataranni PA, Foley JE, Bogardus C, Howard BV, Ravussin E (1995) A high concentration of fasting plasma nonesterified fatty acids is a risk factor for the development of NIDD M. Diabetologia 38:1213-1217

20. Stefan N, Stumvoll M, Bogardus C, Tataranni PA (2003) Elevated plasma nonesterified fatty acids are associated with deterioration of acute insulin response in IGT but not NGT. Am J Physiol Endocrinol Metab 284:E1156-E1161

21. Hills SA, Balkau B, Coppack SW et al (2004) The EGIR-RISC STUDY (The European group for the study of insulin resistance: relationship between insulin sensitivity and cardiovascular disease risk): I. Methodology and objectives. Diabetologia 47:566-570

22. Ferrannini E, Balkau B, Coppack SW et al (2007) Insulin resistance, insulin response, and obesity as indicators of metabolic risk. J Clin Endocrinol Metab 92:2885-2892

23. Mari A, Tura A, Gastaldelli A, Ferrannini E (2002) Assessing insulin secretion by modeling in multiple-meal tests: role of potentiation. Diabetes 51(Suppl 1):S221-S226

24. Mari A, Schmitz O, Gastaldelli A, Oestergaard T, Nyholm B, Ferrannini E (2002) Meal and oral glucose tests for assessment of beta-cell function: modeling analysis in normal subjects. Am $\mathrm{J}$ Physiol Endocrinol Metab 283:E1159-E1166

25. Mari A, Ferrannini E (2008) Beta-cell function assessment from modelling of oral tests: an effective approach. Diabetes Obes Metab 10(Suppl 4):S77-S87

26. Van Cauter E, Mestrez F, Sturis J, Polonsky KS (1992) Estimation of insulin secretion rates from C-peptide levels. Comparison of individual and standard kinetic parameters for C-peptide clearance. Diabetes 41:368-377

27. Stefanovski D, Richey JM, Woolcott O et al (2011) Consistency of the disposition index in the face of diet induced insulin resistance: potential role of FFA. PLoS One 6:e18134
28. Groop LC, Saloranta C, Shank M, Bonadonna RC, Ferrannini E, DeFronzo RA (1991) The role of free fatty acid metabolism in the pathogenesis of insulin resistance in obesity and noninsulindependent diabetes mellitus. J Clin Endocrinol Metab 72:96-107

29. Groop LC, Bonadonna RC, DelPrato S et al (1989) Glucose and free fatty acid metabolism in non-insulin-dependent diabetes mellitus. Evidence for multiple sites of insulin resistance. J Clin Invest 84:205-213

30. Santomauro AT, Boden G, Silva ME et al (1999) Overnight lowering of free fatty acids with Acipimox improves insulin resistance and glucose tolerance in obese diabetic and nondiabetic subjects. Diabetes 48:1836-1841

31. Karpe F, Dickmann JR, Frayn KN (2011) Fatty acids, obesity, and insulin resistance: time for a reevaluation. Diabetes 60:2441-2449

32. Dobbins RL, Chester MW, Daniels MB, McGarry JD, Stein DT (1998) Circulating fatty acids are essential for efficient glucosestimulated insulin secretion after prolonged fasting in humans. Diabetes 7:1613-1618

33. Santini E, Fallahi P, Ferrari SM, Masoni A, Antonelli A, Ferrannini E (2004) Effect of PPAR-gamma activation and inhibition on glucose-stimulated insulin release in INS-1e cells. Diabetes 53(Suppl 3):S79-S83

34. Hellmuth C, Demmelmair H, Schmitt I, Peissner W, Bluher M, Koletzko B (2013) Association between plasma nonesterified fatty acids species and adipose tissue fatty acid composition. PLoS One 8:e74927

35. Byrne CD, Maison P, Halsall D, Martensz N, Hales CN, Wareham NJ (1999) Cross-sectional but not longitudinal associations between non-esterified fatty acid levels and glucose intolerance and other features of the metabolic syndrome. Diabet Med 16:10071015

36. Salgin B, Ong KK, Thankamony A, Emmett P, Wareham NJ, Dunger DB (2012) Higher fasting plasma free fatty acid levels are associated with lower insulin secretion in children and adults and a higher incidence of type 2 diabetes. J Clin Endocrinol Metab 97: 3302-3309

37. Gillum MP, Zhang D, Zhang XM et al (2008) Nacylphosphatidylethanolamine, a gut- derived circulating factor induced by fat ingestion, inhibits food intake. Cell 135:813-824 